E. NAVARRO, R. COMPANY and L. JÓDAR (Valencia)

\title{
BESSEL MATRIX DIFFERENTIAL EQUATIONS: EXPLICIT SOLUTIONS OF INITIAL AND TWO-POINT BOUNDARY VALUE PROBLEMS
}

Abstract. In this paper we consider Bessel equations of the type $t^{2} X^{(2)}(t)$ $+t X^{(1)}(t)+\left(t^{2} I-A^{2}\right) X(t)=0$, where $A$ is an $n \times n$ complex matrix and $X(t)$ is an $n \times m$ matrix for $t>0$. Following the ideas of the scalar case we introduce the concept of a fundamental set of solutions for the above equation expressed in terms of the data dimension. This concept allows us to give an explicit closed form solution of initial and two-point boundary value problems related to the Bessel equation.

1. Introduction. Numerous problems from chemistry, physics and mechanics, both linear and nonlinear, are related to matrix differential equations of the type $t^{2} X^{(2)}(t)+t A(t) X^{(1)}(t)+B(t) X(t)=0$, where $A(t), B(t)$ are matrix-valued functions [8], [10]. This paper is concerned with the Bessel matrix equation

$$
t^{2} X^{(2)}(t)+t X^{(1)}(t)+\left(t^{2} I-A^{2}\right) X(t)=0, \quad t>0,
$$

where $A$ is a matrix in $\mathbb{C}_{n \times n}$, and $X(t)$ is a matrix in $\mathbb{C}_{n \times n}$, for $t>0$. Note that the matrix problem (1.1) may be regarded as a system of coupled Bessel type equations that cannot be transformed into a set of independent equations if the matrix $A$ is not diagonalizable. Standard techniques to study problems related to (1.1) are based on the consideration of the extended first order system

$$
t Z^{\prime}(t)=M(t) Z(t)
$$

where

1991 Mathematics Subject Classification: 33C10, 34A30, 47A60.

Key words and phrases: Bessel matrix equation, fundamental set, closed form solution, boundary value problem, initial value problem. 


$$
M(t)=\left[\begin{array}{cc}
0 & I \\
-t^{2} I+A^{2} & 0
\end{array}\right], \quad Z(t)=\left[\begin{array}{c}
X(t) \\
t X^{\prime}(t)
\end{array}\right] .
$$

Then series solutions for (1.2) may be obtained, and the relationship between the solutions $X(t)$ of (1.1) and $Z(t)$ of (1.2) is given by $X(t)=[I, 0] Z(t)$ (see [4], [13] for details). This technique has two basic drawbacks, first of all it involves an increase of the problem dimension and a lack of explicitness derived from the relationship $X(t)=[I, 0] Z(t)$. Secondly, unlike the scalar case it does not provide a pair of solutions of (1.1) which would allow us to give a closed form of the general solution of (1.1) involving a pair of parameters.

This paper is organized as follows. Section 2 is concerned with some preliminaries that will be used in the following sections. In Section 3 we construct series solutions of problem (1.1) and we propose a closed form of the general solution of (1.1) for the case where the matrix $A$ satisfies the spectral condition

(1.3) For every eigenvalue $z \in \sigma(A), 2 z$ is not an integer, and if $z, w$ belong to $\sigma(A)$ and $z \neq w$, then $z \pm w$ is not an integer.

Here $\sigma(A)$ denotes the set of all eigenvalues of $A$. Finally, in Section 4 we study the boundary value problem

$$
\begin{aligned}
& t^{2} X^{(2)}(t)+t X^{(1)}(t)+\left(t^{2} I-A^{2}\right) X(t)=0, \quad 0<a \leq t \leq b, \\
& M_{11} X(a)+N_{11} X(b)+M_{12} X^{(1)}(a)+N_{12} X^{(1)}(b)=0, \\
& M_{21} X(a)+N_{21} X(b)+M_{22} X^{(1)}(a)+N_{22} X^{(1)}(b)=0,
\end{aligned}
$$

where $M_{i j}, N_{i j}$, for $1 \leq i, j \leq 2$, are matrices in $\mathbb{C}_{n \times n}$.

If $S$ is a matrix in $\mathbb{C}_{m \times n}$, we denote by $S^{+}$its Moore-Penrose pseudoinverse and we recall that an account of uses and properties of this concept may be found in [1].

2. Preliminaries. We begin this section with an algebraic result that provides a finite expression for the solution of a generalized algebraic Lyapunov matrix equation

$$
A_{1}+B_{1} X-X D_{1}=0
$$

where $A_{1}, B_{1}, D_{1}$ and the unknown $X$ are matrices in $\mathbb{C}_{n \times n}$.

Lemma 1. Suppose that matrices $B_{1}$ and $D_{1}$ satisfy the spectral condition

$$
\sigma\left(B_{1}\right) \cap \sigma\left(D_{1}\right)=\emptyset
$$

and let $p(z)=\sum_{k=0}^{n} a_{k} z^{k}$ be such that $p\left(B_{1}\right)=0$. Then the only solution 
$X$ of equation (2.1) is given by

$$
X=\left(\sum_{j=1}^{n} \sum_{h=1}^{j} a_{j} B_{1}^{h-1} A_{1} D_{1}^{j-h}\right)\left(\sum_{j=0}^{n} a_{j} D_{1}^{j}\right)^{-1} .
$$

P r o o f. Under the hypothesis (2.2), equation (2.1) has only one solution [2], [12], and from Corollary 2 of [2], if $X$ is the only solution of (2.1), it follows that

$$
\begin{aligned}
& V=\left[\begin{array}{cc}
B_{1} & A_{1} \\
0 & D_{1}
\end{array}\right]=W\left[\begin{array}{cc}
B_{1} & 0 \\
0 & D_{1}
\end{array}\right] W^{-1}, \\
& W=\left[\begin{array}{cc}
I & X \\
0 & I
\end{array}\right], \quad W^{-1}=\left[\begin{array}{cc}
I & -X \\
0 & I
\end{array}\right] .
\end{aligned}
$$

From (2.4), it follows that

$$
\begin{aligned}
p(V) & =W p\left(\left[\begin{array}{cc}
B_{1} & 0 \\
0 & D_{1}
\end{array}\right]\right) W^{-1} \\
& =W\left[\begin{array}{cc}
p\left(B_{1}\right) & 0 \\
0 & p\left(D_{1}\right)
\end{array}\right] W^{-1}=\left[\begin{array}{cc}
0 & X p\left(D_{1}\right) \\
0 & p\left(D_{1}\right)
\end{array}\right]
\end{aligned}
$$

and taking into account the polynomial calculus there exists a matrix $M$ such that

$$
p(V)=p\left(\left[\begin{array}{cc}
B_{1} & A_{1} \\
0 & D_{1}
\end{array}\right]\right)=\left[\begin{array}{cc}
p\left(B_{1}\right) & M \\
0 & p\left(D_{1}\right)
\end{array}\right]=\left[\begin{array}{cc}
0 & M \\
0 & p\left(D_{1}\right)
\end{array}\right] .
$$

From (2.5) and (2.6) one sees that $X p\left(D_{1}\right)=M$ and from the spectral mapping theorem $\left[3\right.$, p. 569] and (2.2), the matrix $p\left(D_{1}\right)$ is invertible. Thus we have $X=M\left(p\left(D_{1}\right)\right)^{-1}$. On the other hand, considering the powers $V^{j}, j=0,1, \ldots, n$, one finds that the $(i, 2)$ block entry of the operator $V^{j}$, denoted by $V_{i, 2}^{j}$ for $j=1, \ldots, n, i=1,2$, satisfies

$$
V_{1,2}^{j}=B_{1} V_{1,2}^{j-1}+A_{1} V_{2,2}^{j-1}, \quad V_{2,2}^{j}=D_{1}^{j}, \quad V_{1,2}^{0}=0, \quad V_{2,2}^{0}=I .
$$

By multiplying the matrix $V_{1,2}^{j}$ by the coefficient $a_{j}$ for $j=0,1, \ldots, n$ and by addition it follows that the block entry $(1,2)$ of the block matrix $p(V)$ is given by the expression

$$
M=\sum_{j=1}^{n} \sum_{h=1}^{j} a_{j} B_{1}^{h-1} A_{1} D_{1}^{j-h} .
$$

Hence the result is established.

In accordance with the definition given in [6] for a time invariant regular second order matrix differential equation, we introduce the concept of a fundamental set of solutions for equations of the type

$$
Y^{(2)}(t)+P(t) Y^{(1)}(t)+Q(t) Y(t)=0 .
$$


Definition 1. Consider equation (2.7) where $P(t), Q(t)$ are continuous $\mathbb{C}_{n \times n}$-valued functions on an interval $J$ of the real line, and $Y(t) \in \mathbb{C}_{n \times n}$. We say that a pair of solutions $\left\{Y_{1}, Y_{2}\right\}$ is a fundamental set of solutions of (2.7) in the interval $J$ if for any solution $Z$ of (2.7) defined in $J$, there exist matrices $C, D \in \mathbb{C}_{n \times n}$, uniquely determined by $Z$, such that

$$
Z(t)=Y_{1}(t) C+Y_{2}(t) D, \quad t \in J .
$$

The following result provides a useful characterization of a fundamental set of solutions of (2.7) and it may be regarded as an analogue of Liouville's formula for the scalar case.

LEMMA 2. Let $\left\{Y_{1}, Y_{2}\right\}$ be a pair of solutions of (2.7) defined on the interval $J$ and let $W(t)$ be the block matrix function

$$
W(t)=\left[\begin{array}{cc}
Y_{1}(t) & Y_{2}(t) \\
Y_{1}^{(1)}(t) & Y_{2}^{(1)}(t)
\end{array}\right] .
$$

Then $\left\{Y_{1}, Y_{2}\right\}$ is a fundamental set solutions of (2.7) on $J$ if there exists a point $t_{1} \in J$ such that $W\left(t_{1}\right)$ is nonsingular in $\mathbb{C}_{2 n \times 2 n}$. In this case $W(t)$ is nonsingular for all $t \in J$.

Proof. Since $Y_{1}(t)$ and $Y_{2}(t)$ are solutions of $(2.7)$, it follows that $W(t)$ defined by (2.9) satisfies

$$
W^{(1)}(t)=\left[\begin{array}{cc}
0 & I \\
-Q(t) & -P(t)
\end{array}\right] W(t), \quad t \in J .
$$

Thus if $G(t, s)$ is the transition state matrix of (2.10) such that $G(t, t)=I$ [7, p. 598], it follows that $W(t)=G\left(t, t_{1}\right) W\left(t_{1}\right)$ for all $t \in J$. Hence the result is established because $G(t, s)$ is invertible for all $t, s$ in $J$.

Note that in the interval $0<t<\infty$, equation (1.1) takes the form (2.7) with $P(t)=I / t$ and $Q(t)=I-(A / t)^{2}$.

We conclude this section with some recalls concerned with the reciprocal gamma function that may be found in [4, p. 253]. The reciprocal gamma function, denoted by $\Gamma^{-1}(z)=1 / \Gamma(z)$, is an entire function of the complex variable $z$, and thus for any matrix $C \in \mathbb{C}_{n \times n}$, the Riesz-Dunford functional calculus shows that $\Gamma^{-1}(C)$ is a well defined matrix (see Chapter 7 of [3]). If $C$ is a matrix in $\mathbb{C}_{n \times n}$ such that

$$
C+n I \text { is invertible for all integer } n \geq 0
$$

then from [4, p. 253], it follows that

$$
C(C+I)(C+2 I) \ldots(C+n I) \Gamma^{-1}(C+(n+1) I)=\Gamma^{-1}(C) .
$$

Under the condition $(2.11), \Gamma(C)$ is well defined and it is the inverse matrix of $\Gamma^{-1}(C)$. From the properties of the functional calculus $\Gamma^{-1}(C)$ commutes with $C$ and from [3, p. 557], $\Gamma(C)$ and $\Gamma^{-1}(C)$ are polynomials in $C$. 
In particular, if $C$ is a matrix satisfying $(2.11)$, and $\operatorname{Re}(z)>0$ for every eigenvalue $z \in \sigma(C)$, then we have

$$
\Gamma(C)=\int_{0}^{\infty} \exp (-t) \exp ((C-I) \ln t) d t
$$

and this representation of $\Gamma(C)$ coincides with the power series expansion, the Riesz-Dunford formula for $\Gamma(C)[3$, p. 555] and others (see [4, p. 253]). Note that if $C$ satisfies (2.11), from the previous comments and (2.12) we have

$$
\Gamma(C+(n+I))=C(C+I)(C+2 I) \ldots(C+n I) \Gamma(C) .
$$

Note that from (2.13) and (2.14), for matrices $C$ satisfying (2.11) the computation of $\Gamma(C)$ may be performed in an analogous way to the scalar case.

3. Bessel matrix differential equations. Suppose that we are looking for solutions of equation (1.1) of the form

$$
X(t)=\left(\sum_{k \geq 0} C_{k} t^{k}\right) t^{Z}
$$

where $C_{k}$ is a matrix in $\mathbb{C}_{n \times n}, Z \in \mathbb{C}_{n \times n}$ and $t^{Z}=\exp (Z \ln t)$, for $t>0$.

By taking formal derivatives in (3.1), it follows that

$$
\begin{gathered}
X^{(1)}(t)=\sum_{k \geq 0} C_{k}(k I+Z) t^{Z+(k-1) I}, \\
X^{(2)}(t)=\sum_{k \geq 0} C_{k}(k I+Z)(k I+Z-I) t^{Z+(k-2) I} .
\end{gathered}
$$

Assuming the convergence of the series (3.1), (3.2), and substituting into equation (1.1), it follows that

$$
\begin{aligned}
\left\{\sum_{k \geq 0}\left[C_{k}(k I+Z)(k I+Z-I)+C_{k}(k I+Z)-A^{2} C_{k}\right] t^{k}\right. & \\
& \left.+\sum_{k \geq 2} C_{k-2} t^{k}\right\} t^{Z}=0 .
\end{aligned}
$$

By equating to the zero matrix the coefficient of each power $t^{k}$ appearing in (3.3), it follows that the matrices $C_{k}$ must satisfy

$$
\begin{gathered}
C_{0} Z(Z-I)+C_{0} Z-A^{2} C_{0}=C_{0} Z^{2}-A^{2} C_{0}=0, \\
C_{1}(Z+I) Z+C_{1}(Z+I)-A^{2} C_{1}=C_{1}(Z+I)^{2}-A^{2} C_{1}=0, \\
C_{k}(k I+Z)^{2}-A^{2} C_{k}=-C_{k-2}, \quad k \geq 2 .
\end{gathered}
$$

Let $Z$ be a matrix in $\mathbb{C}_{n \times n}$ and let $C_{0}$ be an invertible matrix in $\mathbb{C}_{n \times n}$ such 
that

$$
Z=C_{0}^{-1} A C_{0}
$$

Then

$$
\sigma(A)=\sigma(Z), \quad Z^{2}=C_{0}^{-1} A^{2} C_{0}, \quad C_{0} Z^{2}-A^{2} C_{0}=0 .
$$

Given the matrix $Z$ defined by (3.7), from (1.3) and Lemma 1, the only solution for $C_{1}$ of the matrix equation

$$
C_{1}(Z+I)^{2}-A^{2} C_{1}=0
$$

is the zero matrix $C_{1}=0$. From (3.6) it follows that $C_{2 m+1}=0$ for $m \geq 0$. In order to determine the matrix coefficients $C_{2 m}$, let $p(z)$ be an annihilating polynomial of the matrix $A^{2}$,

$$
p(z)=\sum_{j=0}^{n} a_{j} z^{j}, \quad p\left(A^{2}\right)=0 .
$$

Under the hypothesis (1.3) it follows that $\sigma\left((k I+Z)^{2}\right) \cap \sigma\left(A^{2}\right)=\emptyset$ for $k \geq 1$, and from Lemma 1 , the only solution $C_{2 m}$ of the equation

$$
A^{2} C_{2 m}-C_{2 m}(2 m I+Z)^{2}=C_{2 m-2}, \quad m \geq 1,
$$

is given by

$$
\begin{aligned}
& C_{2 m}= \\
& -\left(\sum_{j=1}^{n} \sum_{h=1}^{j} a_{j} A^{2 h-2} C_{2 m-2}(2 m I+Z)^{2(j-h)}\right)\left(\sum_{j=1}^{n} a_{j}(2 m I+Z)^{2 j}\right)^{-1} .
\end{aligned}
$$

Note that once we choose the matrices $C_{0}$ and $Z$, all the matrix coefficients $C_{2 m}$ for $m \geq 1$ are determined by (3.11).

Now we are concerned with the proof of the convergence of the series

$$
X\left(t, Z, C_{0}\right)=\left(\sum_{m \geq 0} C_{2 m} t^{2 m}\right) t^{Z} .
$$

The generalized power series (3.12) is convergent for $t>0$ if the power series

$$
U\left(t, Z, C_{0}\right)=\sum_{m \geq 0} C_{2 m} t^{2 m},
$$

is convergent for $t>0$.

If $B$ is a matrix in $\mathbb{C}_{n \times n}$ and $B^{H}$ denotes the conjugate transpose of $B$, we denote by $\|B\|$ its spectral norm, defined to be the maximum of the set $\left\{|z|^{1 / 2}: z \in \sigma\left(B^{H} H\right)\right\}$. Taking norms in (3.10), for large values of $m$ it follows that

$$
\begin{aligned}
\left\|C_{2 m-2}\right\| & =\left\|C_{2 m}(2 m I+Z)^{2}-A^{2} C_{2 m}\right\| \\
& \geq\left|\left\|C_{2 m}(2 m I+Z)^{2}\right\|-\left\|A^{2} C_{2 m}\right\|\right|
\end{aligned}
$$




$$
\geq\left\|C_{2 m}\right\|\left(4 m^{2}-4 m\|Z\|-\left\|Z^{2}\right\|-\left\|A^{2}\right\|\right) .
$$

Hence

$$
\frac{\left\|C_{2 m}\right\||t|^{2 m}}{\left.\left\|C_{2 m-2}\right\| t\right|^{2 m-2}} \leq \frac{|t|^{2}}{4 m^{2}-4 m\|Z\|-\left\|Z^{2}\right\|-\left\|A^{2}\right\|}
$$

and this proves the absolute convergence of the series (3.13) for $t>0$.

Now we are going to find a second solution of (1.1) of the form

$$
X\left(t,-Z, C_{0}\right)=\left(\sum_{k \geq 0} C_{k}^{*} t^{k}\right) t^{-Z}=U\left(t,-Z, C_{0}\right) t^{-Z}
$$

where $C_{0}$ is the matrix satisfying (3.7). In an analogous way to the construction of $X\left(t, Z, C_{0}\right)$, it is straightforward to show that the matrices $C_{k}^{*}$ appearing in (3.15), for $k \geq 0$, with $C_{0}^{*}=C_{0}$, must satisfy the equations

$$
\begin{gathered}
C_{0}^{*} Z^{2}-A^{2} C_{0}^{*}=0, \quad C_{1}^{*}(I-Z)^{2}-A^{2} C_{1}^{*}=0, \\
C_{k}^{*}(k I-Z)^{2}-A^{2} C_{k}^{*}=-C_{k-2}^{*}, \quad k \geq 2 .
\end{gathered}
$$

From the hyphothesis (1.3), (3.16) and Lemma 1, it follows that $C_{1}^{*}=$ $C_{2 m+1}^{*}=0$, and, for $m \geq 1$,

$$
=\left(\sum_{j=1}^{n} \sum_{h=1}^{j} a_{j} A^{2 h-2} C_{2 m-2}^{*}(2 m I-Z)^{2(j-h)}\right)\left(\sum_{j=0}^{n} a_{j}(2 m I-Z)^{2 j}\right)^{-1} .
$$

The proof of the absolute convergence of the series

$$
U\left(t,-Z, C_{0}\right)=\sum_{m \geq 0} C_{2 m}^{*} t^{2 m}
$$

for $t>0$ is analogous to the previous proof for $U\left(t, Z, C_{0}\right)$.

Now we are going to prove that for any invertible matrices $C_{0}$ and $Z$ satisfying (3.7), the pair defined by $X\left(t, Z, C_{0}\right)$ and $X\left(t,-Z, C_{0}\right)$ is a fundamental set of solutions of (1.1) in $0<t<\infty$. The Wroński block matrix function associated with this pair and defined by (2.9) takes the form

$$
\begin{aligned}
& (3.19) \quad W(t) \\
& =\left[\begin{array}{cc}
U\left(t, Z, C_{0}\right) t^{Z} & U\left(t,-Z, C_{0}\right) t^{-Z} \\
U^{(1)}\left(t, Z, C_{0}\right) t^{Z}+U\left(t, Z, C_{0}\right) Z t^{Z-I} & U^{(1)}\left(t,-Z, C_{0}\right) t^{-Z}-U\left(t,-Z, C_{0}\right) Z t^{-Z-I}
\end{array}\right] \\
& =\left[\begin{array}{cc}
I & 0 \\
0 & t^{-1} I
\end{array}\right] T(t)\left[\begin{array}{cc}
t^{Z} & 0 \\
0 & t^{-Z}
\end{array}\right]
\end{aligned}
$$

where

(3.20) $T(t)$

$$
=\left[\begin{array}{cc}
U\left(t, Z, C_{0}\right) & U\left(t,-Z, C_{0}\right) \\
U^{(1)}\left(t, Z, C_{0}\right) t+U\left(t, Z, C_{0}\right) Z & U^{(1)}\left(t,-Z, C_{0}\right) t-U\left(t,-Z, C_{0}\right) Z
\end{array}\right] .
$$


From (3.19) it is clear that $W(t)$ is invertible if and only if $T(t)$ is invertible. Note that $T(t)$ is a continuous $\mathbb{C}_{2 n \times 2 n}$-valued function defined in the interval $[0, \infty)$. Since $T(0)$ is the matrix

$$
T(0)=\left[\begin{array}{cc}
C_{0} & C_{0} \\
C_{0} Z & -C_{0} Z
\end{array}\right],
$$

it is invertible because of the invertibility of $C_{0}$, Lemma 1 of [5] and the fact that

$$
-C_{0} Z-\left(C_{0} Z\right) C_{0}^{-1} C_{0}=-2 C_{0} Z \text { is invertible. }
$$

From the invertibility of $T(0)$ and the Perturbation Lemma [9, p. 32], there exists a positive number $t_{1}$ such that $T(t)$ is invertible in $\left[0, t_{1}\right]$. This proves the invertibility of $W\left(t_{1}\right)$ and from Lemma 2 the pair $\left\{X\left(\cdot, Z, C_{0}\right)\right.$, $\left.X\left(\cdot,-Z, C_{0}\right)\right\}$ is a fundamental set of solutions of equation (1.1) in $0<t<$ $\infty$. From the previous comments the following result has been proved:

TheOREM 1. Let $C_{0}$ and $Z$ be invertible matrices in $\mathbb{C}_{n \times n}$ and let $A$ be a matrix in $\mathbb{C}_{n \times n}$ satisfying (1.3). Then the pair $\left\{X\left(\cdot, Z, C_{0}\right), X\left(\cdot,-Z, C_{0}\right)\right\}$ defined by (3.11), (3.12), (3.15), (3.17), (3.18) is a fundamental set of solutions of the Bessel equation (1.1) in $0<t<\infty$. The general solution of (1.1) in $0<t<\infty$ is given by

$$
X(t)=X\left(t, Z, C_{0}\right) P+X\left(t,-Z, C_{0}\right) Q, \quad P, Q \in \mathbb{C}_{n \times n} .
$$

The unique solution of (1.1) satisfying the initial conditions $X(a)=E$, $X^{(1)}(a)=F$, with $0<a<\infty$, is given by (3.21) where

$$
\left[\begin{array}{l}
P \\
Q
\end{array}\right]=(W(a))^{-1}\left[\begin{array}{l}
E \\
F
\end{array}\right]
$$

and $W(a)$ is defined by (3.19).

Rem ark 1. If we consider the Bessel equation (1.1) with vector-valued unknown $X(t)$, then considering the fundamental set of solutions constructed in Theorem 1, the general solution of the vector problem (1.1) is given by (3.21) upon replacing the matrices $P, Q$, by arbitrary vectors $P, Q$ in $\mathbb{C}_{n \times 1}$.

Now we are interested in showing that for the case where the matrix $A$ is diagonalizable and satisfies (1.3), the fundamental set of solutions constructed in Theorem 1 coincides with the well known one for the scalar case when $n=1$, given in terms of the Bessel functions of the first kind.

Let $A$ be a diagonalizable matrix satisfying (1.3) and let $C_{0}$ be a basis of $\mathbb{C}_{n \times 1}$ composed of eigenvectors of $A$. If $\sigma(A)=\left\{\lambda_{1}, \ldots, \lambda_{n}\right\}$, and $Z=$ $\operatorname{diag}\left(\lambda_{s}: 1 \leq s \leq n\right)$, then we have

$$
Z=C_{0}^{-1} A C_{0} .
$$


On the other hand, if we denote by $B^{(i)}$ the $i$ th column of the matrix $B \in \mathbb{C}_{n \times n}$, taking the $i$ th column in both members of equation (3.6), it follows that

$$
\left(\left(k+\lambda_{s}\right)^{2} I-A^{2}\right) C_{k}^{(s)}=-C_{k-2}^{(s)}, \quad 1 \leq s \leq n, k \geq 2 .
$$

Note that we may write the matrix $\left(m+\frac{1}{2} \lambda_{s}\right)^{2} I-A^{2}$ in the form

$$
\begin{gathered}
\left(m+\frac{1}{2} \lambda_{s}\right)^{2} I-A^{2}=\left(\left(m+\frac{1}{2} \lambda_{s}\right) I+A\right)\left(\left(m+\frac{1}{2} \lambda_{s}\right) I-A\right. \\
=\left(m I+\frac{1}{2}\left(\lambda_{s} I+A\right)\right)\left(m I+\frac{1}{2}\left(\lambda_{s} I-A\right)\right) \\
=\left(m I+B_{s}\right)\left(m I+D_{s}\right), \\
B_{s}=\frac{1}{2}\left(\lambda_{s} I+A\right), \quad D_{s}=\frac{1}{2}\left(\lambda_{s} I-A\right) .
\end{gathered}
$$

Considering (3.22) for even integers $k=2 m$, we have

$$
\begin{aligned}
C_{2 m}^{(s)} & =\frac{(-1)^{m}}{2^{2 m}} \prod_{j=1}^{m}\left(\left(j+\frac{1}{2} \lambda_{s}\right)^{2} I-A^{2}\right)^{-1} C_{0}^{(s)} \\
& =\frac{(-1)^{m}}{2^{2 m}} \prod_{j=1}^{m}\left(j I+D_{s}\right)^{-1}\left(j I+B_{s}\right)^{-1} C_{0}^{(s)}, \quad 1 \leq s \leq n .
\end{aligned}
$$

Now consider the new basis of eigenvectors of $A$ defined by the matrix $K_{0}$ whose $s$ th column is given by

$$
C_{0}^{(s)}=2^{\lambda_{s}} \Gamma\left(D_{s}+I\right) \Gamma\left(B_{s}+I\right) K_{0}^{(s)}, \quad 1 \leq s \leq n .
$$

Note that from (1.3) and (3.23), the matrices $\Gamma\left(D_{s}+I\right)$ and $\Gamma\left(B_{s}+I\right)$ are invertible and commute with $A$. This proves that the columns of $K_{0}$ define a basis of eigenvectors of $A$ satisfying

$$
Z=K_{0}^{-1} A K_{0} \text {. }
$$

The corresponding equations (3.24) for $K_{2 m}^{(s)}$ satisfy

$K_{2 m}^{(s)}=\frac{(-1)^{m}}{2^{2 m}} \prod_{j=1}^{m}\left(j I+D_{s}\right)^{-1}\left(j I+B_{s}\right)^{-1} \Gamma^{-1}\left(B_{s}+I\right) \Gamma^{-1}\left(D_{s}+I\right) C_{0}^{(s)} 2^{-\lambda_{s}}$.

Taking into account (2.14) and the fact that $j I+B_{s}$ and $j I+D_{s}$ commute shows that

$K_{2 m}^{(s)}=\frac{(-1)^{m}}{2^{2 m}} \Gamma^{-1}\left(D_{s}+(m+1) I\right) \Gamma^{-1}\left(B_{s}+(m+1) I\right) C_{0}^{(s)} 2^{-\lambda_{s}}, \quad 1 \leq s \leq n$.

In matrix form the above expression may be written as

$$
\begin{gathered}
K_{2 m}=\frac{(-1)^{m}}{2^{2 m}} L_{2 m} 2^{-Z}, \\
L_{2 m}^{(s)}=\Gamma^{-1}\left(D_{s}+(m+1) I\right) \Gamma^{-1}\left(B_{s}+(m+1) I\right) C_{0}^{(s)},
\end{gathered}
$$


and $X\left(t, Z, K_{0}\right)$ takes the form

$$
\begin{aligned}
X\left(t, Z, K_{0}\right) & =\left(\sum_{m \geq 0} K_{2 m} t^{2 m}\right) t^{Z} \\
& =\left(\sum_{m \geq 0} \frac{(-1)^{m}}{2^{2 m}} L_{2 m} t^{2 m}\right)(t / 2)^{Z}, \quad t>0 .
\end{aligned}
$$

In an analogous way, if we denote by $B_{s}^{*}$ and $D_{s}^{*}$ the matrices

$$
\begin{aligned}
& B_{s}^{*}=\frac{1}{2}\left(A-\lambda_{s} I\right), \\
& D_{s}^{*}=\frac{1}{2}\left(-A-\lambda_{s} I\right), \quad 1 \leq s \leq n,
\end{aligned}
$$

and

$$
\begin{gathered}
K_{2 m}^{*}=\frac{(-1)^{m}}{2^{2 m}} L_{2 m}^{*} 2^{Z}, \\
L_{2 m}^{*(s)}=\Gamma^{-1}\left(D_{s}^{*}+(m+1) I\right) \Gamma^{-1}\left(B_{s}^{*}+(m+1) I\right) C_{0}^{(s)},
\end{gathered}
$$

then

$$
\begin{aligned}
X\left(t,-Z, K_{0}\right) & =\left(\sum_{m \geq 0} K_{2 m}^{*} t^{2 m}\right) t^{-Z} \\
& =\left(\sum_{m \geq 0} \frac{(-1)^{m}}{2^{2 m}} L_{2 m}^{*} t^{2 m}\right)(t / 2)^{-Z}, \quad t>0 .
\end{aligned}
$$

Thus for the case where $A$ is diagonalizable and $\sigma(A)=\left\{\lambda_{s}: 1 \leq s \leq n\right\}$, Theorem 1 provides the fundamental set of solutions in $0<t<\infty$, defined by $X\left(\cdot, Z, K_{0}\right)$ and $X\left(\cdot,-Z, K_{0}\right)$.

Now we show that for the scalar case, when $A=\nu$ is a complex number such that $2 \nu$ is not an integer, which is the condition (1.3) for the case $n=1$, the fundamental set of solutions of (1.1) given by (3.28) and (3.31) coincides with the Bessel functions of the first kind $J_{\nu}(x)$ and $J_{-\nu}(x)$, respectively. Note that for the scalar case we have

$$
\begin{gathered}
A=Z=\nu, \quad C_{0}=1, \\
B_{1}=\frac{1}{2}(\nu+\nu)=\nu, \quad B_{1}^{*}=\frac{1}{2}(\nu-\nu)=0, \\
D_{1}=\frac{1}{2}(\nu-\nu)=0, \quad D_{1}^{*}=\frac{1}{2}(-\nu-\nu)=-\nu, \\
\Gamma^{-1}\left(B_{1}+(m+1) I\right)=\Gamma^{-1}(\nu+m+1), \\
\Gamma^{-1}\left(D_{1}+(m+1) I\right)=\Gamma^{-1}(m+1)=1 / m !, \\
L_{2 m}=\frac{1}{m ! \Gamma(\nu+m+1)}, \\
L_{2 m}^{*}=\frac{1}{m ! \Gamma(-\nu+m+1)},
\end{gathered}
$$




$$
\begin{aligned}
K_{0} & =K_{0}^{(1)}=2^{-Z} \Gamma^{-1}\left(B_{1}+I\right) \Gamma^{-1}\left(D_{1}+I\right) \\
& =2^{-\nu} \Gamma^{-1}(\nu+1) 0 !=2^{-\nu} \Gamma^{-1}(\nu+1), \\
K_{0}^{*} & =K_{0}^{*(1)}=2^{Z} \Gamma^{-1}\left(B_{1}^{*}+I\right) \Gamma^{-1}\left(D_{1}^{*}+I\right) \\
& =2^{\nu} \Gamma^{-1}(1) \Gamma^{-1}(-\nu+1)=2^{\nu} \Gamma^{-1}(-\nu+1) .
\end{aligned}
$$

Hence for the scalar case with $A=\nu$ such that $2 \nu$ is not an integer, taking $K_{0}$ and $K_{0}^{*}$ defined by (3.32), it follows that the fundamental set of solutions of (1.1) given by (3.28), (3.31) is

$$
X\left(t, \nu, K_{0}\right)=J_{\nu}(t), \quad X\left(t,-\nu, K_{0}\right)=J_{-\nu}(t), \quad t>0,
$$

where $J_{\nu}(t)$ and $J_{-\nu}(t)$ denote the Bessel functions of the first kind of order $\nu$.

4. Boundary value problems. Under the hypotheses and notation of Section 3, let $X\left(t, Z, C_{0}\right), X\left(t,-Z, C_{0}\right)$ be a fundamental set of solutions of (1.1), constructed for matrices $Z$ and $C_{0}$ satisfying (3.7). Taking into account the expression (3.21) for the general solution of (1.1) in $t>0$, its derivative is

$$
\begin{aligned}
X^{(1)}(t)= & X^{(1)}\left(t, Z, C_{0}\right) P+X^{(1)}\left(t,-Z, C_{0}\right) Q \\
= & \left(U^{(1)}\left(t, Z, C_{0}\right) t^{Z}+U\left(t, Z, C_{0}\right) Z t^{Z-I}\right) P \\
& +\left(U^{(1)}\left(t,-Z, C_{0}\right) t^{-Z}-U\left(t,-Z, C_{0}\right) Z t^{-Z-I}\right) Q,
\end{aligned}
$$

where $U\left(t, Z, C_{0}\right), U\left(t,-Z, C_{0}\right)$ are defined by (3.13) and (3.18), respectively, and $P, Q$ are arbitrary matrices in $\mathbb{C}_{n \times n}$.

If we impose on the general solution $X(t)$ of (1.1), described by (3.21), the boundary value conditions of (1.4), then from (3.21) and (4.1), it follows that problem (1.4) is solvable if and only if the algebraic system

$$
S\left[\begin{array}{l}
P \\
Q
\end{array}\right]=0
$$

is compatible, where $S=\left(S_{i j}\right)_{1 \leq i, j \leq 2}$ is the block matrix whose entries are

$$
\begin{aligned}
S_{i 1}= & M_{i 1} U\left(a, Z, C_{0}\right) a^{Z}+N_{i 1} U\left(b,-Z, C_{0}\right) b^{Z} \\
& +M_{i 2}\left(U^{(1)}\left(a, Z, C_{0}\right) a^{Z}+U\left(a, Z, C_{0}\right) Z a^{Z-I}\right) \\
& +N_{i 2}\left(U^{(1)}\left(b, Z, C_{0}\right) b^{Z}+U\left(b, Z, C_{0}\right) Z b^{Z-I}\right), \quad i=1,2 \\
S_{i 2}= & M_{i 1} U\left(a,-Z, C_{0}\right) a^{-Z}+N_{i 1} U\left(b,-Z, C_{0}\right) b^{-Z} \\
& +M_{i 2}\left(U^{(1)}\left(a,-Z, C_{0}\right) a^{-Z}-U\left(a,-Z, C_{0}\right) Z a^{-Z-I}\right) \\
& +N_{i 2}\left(U^{(1)}\left(b,-Z, C_{0}\right) b^{-Z}-U\left(b,-Z, C_{0}\right) Z b^{-Z-I}\right), \quad i=1,2 .
\end{aligned}
$$

Thus the boundary value problem (4.1) is solvable if and only if the matrix $S$ defined by (4.3)-(4.4) is singular. Under this condition, from Theorem 2.3.2 
of $[11$, p. 24], the general solution of the algebraic system (4.2) is given by

$$
\left[\begin{array}{l}
P \\
Q
\end{array}\right]=S^{+} S G, \quad G \in \mathbb{C}_{2 n \times n} .
$$

Hence the general solution of problem (1.3), under the hypothesis of singularity for the matrix $S$, is given by (3.21) where the matrices $P, Q$ are given by (4.5) for an arbitrary matrix $G$ in $\mathbb{C}_{2 n \times n}$.

Hence the following result has been established:

TheOREM 2. Under the hypotheses and notation of Theorem 1, let $S$ be the block matrix defined by (4.3)-(4.4) and associated with the fundamental set $\left\{X\left(\cdot, Z, C_{0}\right), X\left(\cdot,-Z, C_{0}\right)\right\}$. Then the boundary value problem (1.3) is solvable if and only if $S$ is singular. Under this condition the general solution of (1.3) is given by (3.21), where $P, Q$ are matrices in $\mathbb{C}_{n \times n}$ given by (4.5).

Acknowledgements. This paper was supported by the D.G.I.C.Y.T. grant PS90-140 and the NATO grant CRG 900040.

\section{References}

[1] S. L. Campbell and C. D. Meyer Jr., Generalized Inverses of Linear Transformations, Pitman, London, 1979.

[2] C. Davis and P. Rosenthal, Solving linear operator equations, Canad. J. Math. 26 (6) (1974), 1384-1389.

[3] N. Dunford and J. Schwartz, Linear Operators, Part I, Interscience, New York, 1957.

[4] E. Hille, Lectures on Ordinary Differential Equations, Addison-Wesley, 1969.

[5] L. Jódar, Explicit expressions for Sturm-Liouville operator problems, Proc. Edinburgh Math. Soc. 30 (1987), 301-309.

[6] - Explicit solutions for second order operator differential equations with two boundary value conditions, Linear Algebra Appl. 103 (1988), 35-53.

[7] T. Kailath, Linear Systems, Prentice-Hall, Englewood Cliffs, N.J., 1980.

[8] H. B. Keller and A. W. Wolfe, On the nonunique equilibrium states and buckling mechanism of spherical shells, J. Soc. Indust. Appl. Math. 13 (1965), 674-705.

[9] J. M. Ortega, Numerical Analysis. A Second Course, Academic Press, New York, 1972 .

[10] S. V. Parter, M. L. Stein and P. R. Stein, On the multiplicity of solutions of a differential equation arising in chemical reactor theory, Tech. Rep. 194, Dept. of Computer Sciences, Univ. of Wisconsin, Madison, 1973.

[11] C. R. Rao and S. K. Mitra, Generalized Inverses of Matrices and its Applications, Wiley, New York, 1971.

[12] M. Rosenblum, On the operator equation $B X-X A=Q$, Duke Math. J. 23 (1956), 263-269. 
[13] E. Weinmüller, A difference method for a singular boundary value problem of second order, Math. Comp. 42 (166) (1984), 441-464.

ENRIQUE NAVARRO, RAFAEL COMPANY AND LUCAS JÓDAR

DEPARTAMENTO DE MATEMÁTICA APLICADA

UNIVERSIDAD POLITÉCNICA DE VALENCIA

P.O. BOX 22.012

46022 VALENCIA, SPAIN

Received on 21.3.1992 